\title{
Non-vaccine serotype pneumococcal carriage in healthy infants in South Africa following introduction of the 13-valent pneumococcal conjugate vaccine
}

\author{
Z Skosana, ${ }^{1}$ BSc Hons, MSc; A von Gottberg, ${ }^{2,3}$ MB BCh, PhD; S Olorunju, ${ }^{4} \mathrm{PhD} ;$ T Mohale, ${ }^{2}$ BSc Hons, MSc; M du Plessis, ${ }^{2,3} \mathrm{PhD}$ \\ T Adams, ${ }^{5}$ Nat Dip Med Tech; N Mbelle, ${ }^{1,6} \mathrm{MB}$ BCh, PhD \\ ${ }^{1}$ Department of Medical Microbiology, School of Medicine, Faculty of Health Sciences, University of Pretoria, South Africa \\ ${ }^{2}$ Centre for Respiratory Diseases and Meningitis, National Institute for Communicable Diseases, National Health Laboratory Service, \\ Johannesburg, South Africa \\ ${ }^{3}$ School of Pathology, Faculty of Health Sciences, University of the Witwatersrand, Johannesburg, South Africa \\ ${ }^{4}$ South African Medical Research Council, Gauteng, South Africa \\ ${ }^{5}$ Department of Clinical Microbiology and Infectious Diseases, Chris Hani Baragwanath Academic Hospital and National Health Laboratory \\ Service, Johannesburg, South Africa \\ ${ }^{6}$ Department of Medical Microbiology, Tshwane Academic Division, National Health Laboratory Service, Pretoria, South Africa
}

Corresponding author: Z Skosana (zoteeskay4@gmail.com)

\begin{abstract}
Background. Pneumococcal carriage studies provide a baseline for measuring the impact of pneumococcal conjugate vaccines (PCVs). The advent of conjugate vaccines has led to reductions in vaccine serotypes (VTs) in pneumococcal carriage. However, increasing non-vaccine serotypes (NVTs) remain a significant concern, necessitating continued surveillance of serotypes in the 13-valent PCV vaccine (PCV13) era. Objectives. To investigate pneumococcal carriage, serotype distribution and risk factors for pneumococcal colonisation among children presenting for routine immunisation at two clinics in Gauteng Province, South Africa (SA), 10 years after PCV introduction into the SA Expanded Programme on Immunisation (EPI-SA).

Methods. Nasopharyngeal swabs were collected from 322 healthy children aged between 6 weeks and 5 years at two clinic centres in 2014 and 2016. Demographic data, risk factors for colonisation and vaccination details were recorded. The pneumococcal isolates were serotyped and tested for antimicrobial susceptibility.

Results. Pneumococci were isolated from 138/316 healthy children (43.7\%) presenting for routine immunisation at two clinics. The median age was 8.3 months and the age range 1.4 months - 5 years. Carriage varied across the age groups: 6 - 14 weeks $35.5 \%, 9$ months $27.5 \%, 18$ months $21.7 \%$, and 5 years $15.2 \%$. Risk factors significantly associated with pneumococcal colonisation included young age ( 9 18 months (odds ratio OR 3.5; 95\% confidence interval (CI) 1.9 - 5.9), type of dwelling (single room (OR 8.1; 95\% CI 1.3 - 52.3) or informal dwelling (OR 2.4; 95\% CI 1.2 - 4.5)) and Haemophilus influenzae carriage (OR 5.6; 95\% CI 0.6 - 2.5). Of the 26 serotypes detected, 19F $(10 / 121 ; 8.3 \%)$ was the most frequent. The most frequent NVTs were 23B $(16 / 121 ; 13.2 \%), 15 \mathrm{~B} / \mathrm{C}(14 / 121 ; 11.6 \%)$ and $35 \mathrm{~B}(11 / 121 ; 8.2 \%)$. Children aged 9 months carried the highest proportion of NVTs (33/101; 32.7\%). Penicillin non-susceptibility was observed in 20 NVT isolates (20/36; 55.6\%) and $2 \mathrm{VT}$ isolates (2/36; 5.6\%).

Conclusions. The pneumococcal carriage prevalence described in our study varied across the age groups and was lower compared with other African studies that looked at pneumococcal carriage post PCV. The study gave insight into the common NVTs encountered at two immunisation clinics in Gauteng. Given that pneumococcal carriage precedes disease, common colonisers such as $15 \mathrm{~B} / \mathrm{C}$ and $35 \mathrm{~B}$ may be sufficiently prevalent in carriage for expansion to result in significant disease replacement.
\end{abstract}

S Afr Med J 2021;111(2):143-148. https://doi.org/10.7196/SAMJ.2021.v111i2.14626

Pneumococcal carriage precedes pneumococcal disease, ${ }^{[1]}$ so patients with invasive pneumococcal disease (IPD) have similar serotypes to those present in the nasopharynx during carriage. ${ }^{[2]}$ The prevalence of pneumococcal carriage has been described as generally high in Africa, particularly in young children, but considerable variation between countries exists. ${ }^{[3]}$ Some studies done in Africa reported a prevalence of $>70 \%$ pneumococcal carriage in children $<5$ years of age before the use of conjugate vaccines. ${ }^{[4,5]}$

Pneumococcal conjugate vaccines (PCVs) have been widely used in high-income countries and provide effective protection against vaccine serotypes (VTs). Recently there has been an increase in the use of PCVs in low- and middle-income countries. ${ }^{[6]}$ PCVs are currently part of the South African (SA) Expanded Programme on
Immunisation (EPI-SA). ${ }^{[7]}$ In 2009, SA was the first African country to introduce the 7-valent PCV (PCV7) for infants at 6,14 and 36 weeks of age, with a novel schedule aligned to the World Health Organization's recommended EPI. ${ }^{[7,8]}$ In 2011, PCV7 was replaced by PCV $13,{ }^{[8]}$ with a catch-up programme for children aged $<2$ years and 6 months. ${ }^{[8]}$

Although the introduction of PCVs caused a decline in the prevalence of VTs in children, pneumococcal carriage remains unchanged because of the concomitant increase in non-vaccine serotypes (NVTs). ${ }^{[3,9]}$ Some of the most frequently carried NVTs are $6 \mathrm{C}, 11 \mathrm{~A}, 15 \mathrm{~A} / \mathrm{B} / \mathrm{C}, 21,22 \mathrm{~F}, 23 \mathrm{~A} / \mathrm{B}$ and $35 \mathrm{~F}^{[10]}$ In addition to direct protection and prevention of pneumococcal disease in vaccinated children, unvaccinated children benefit from reductions of 
pneumococcal disease by the reductions of pneumococcal carriage in those who have been vaccinated, leading to reduced transmission. ${ }^{[11]}$ Surveillance of the prevalence of pneumococcal serotypes in a population is important, to study the impact of PCVs. ${ }^{[10]}$

\section{Objectives}

In this study, we investigated pneumococcal carriage, risk factors associated with colonisation, and the NVTs frequently encountered in infants and children presenting for routine immunisation at two clinics in Gauteng Province, SA, 10 years after PCV introduction into the EPI-SA.

\section{Methods}

\section{Study design and setting}

The study was conducted in the Department of Medical Microbiology, University of Pretoria/National Health Laboratory Service (NHLS), SA.

\section{Study population}

The study population comprised healthy children aged $\sim 6,10$ and 14 weeks, 9 and 18 months, and 5 years presenting for routine immunisation at the KT Motubatse Clinic and the Zola Community Clinic in Gauteng. Sampling took place from June to December 2014 at the KT Motubatse Clinic and from June to December 2016 at the Zola Community Clinic. Nasopharyngeal swabs (CalgiSwab type 1; Spectrum, USA) were collected from 322 children whose parents or guardians had signed written informed consent in their preferred language. Epidemiological data were obtained using a questionnaire that included demographic characteristics, the number of siblings aged $<5$ years who lived in the house, whether the participants attended a day-care centre, smoking habits of family members who lived with the participants, and medical and vaccination history. In addition, the parents/guardians accompanying the participants were asked whether the child had had any illnesses during the past 3 months.

\section{Laboratory procedures}

The swabs were placed in skim milk-tryptone-glucose-glycerin (STGG) and selectively cultured on $5 \%$ sheep blood agar (Diagnostic Media Products, NHLS, SA) containing $5 \mathrm{mg} / \mathrm{mL}$ gentamicin. The agar plates were incubated in $5 \%$ carbon dioxide overnight at $37^{\circ} \mathrm{C}$ to isolate Streptococcus pneumoniae, Staphylococcus aureus, Moraxella catarrhalis, Corynebacterium pseudodiptheriticum and Haemophilus influenzae. S. pneumoniae was identified using colony morphology, alpha haemolysis on blood agar and susceptibility to optochin (5 $\mu \mathrm{g}$; Becton Dickinson Microbiology Systems, USA). The isolates were tested for antimicrobial susceptibility to chloramphenicol, tetracycline, cotrimoxazole, erythromycin, clindamycin and rifampicin (Davies Diagnostics, UK) by disc diffusion (KirbyBauer disc method). Oxacillin discs $(1 \mu \mathrm{g})$ (Davies Diagnostics) were used to screen for penicillin susceptibility. Oxacillin-resistant isolates were further tested using the E-test (Epsilometer test; BioMérieux, USA) (for penicillin and cefotaxime) to determine the minimum inhibitory concentration. The results were interpreted using the 2014 Clinical Laboratory Standards Institute guidelines. ${ }^{[12]}$ Multidrug resistance was defined as non-susceptibility to three or more classes of antimicrobials. Oral penicillin breakpoints were used for the interpretation of penicillin results, i.e. susceptible $\leq 0.06 \mu \mathrm{g} /$ $\mathrm{mL}$, intermediately resistant $0.12-1 \mu \mathrm{g} / \mathrm{mL}$ and resistant $\geq 2 \mu \mathrm{g} /$ $\mathrm{mL}$. Intermediately resistant and resistant isolates were defined as non-susceptible. Of the $138 \mathrm{~S}$. pneumoniae carriage isolates, 121 $(87.7 \%)$ retained viability and were serotyped with the Quellung method using serotype-specific antisera (Statens Serum Institute, Denmark) at the Centre for Respiratory Diseases and Meningitis, National Institute for Communicable Diseases. The isolates were also serotyped by triplex real-time polymerase chain reaction assay containing 38 sets of serogroup/serotype-specific primer pairs.

\section{Statistical analysis}

All data were captured in Epi Info 7 (Centers for Disease Control, USA) and exported to Microsoft Excel, 2016 version (Microsoft, USA). Data were analysed using Stata v15 (StataCorp, USA) and descriptive statistics including proportions and associated 95\% confidence intervals (CIs). Potential risk factors including age, daycare centre attendance, smoking habits of family members who lived with participants, illnesses in the past 3 months, method of cooking, carriage of $S$. aureus and $H$. influenzae, vaccination and type of dwelling were tested by cross-tabulation to assess association between variables, and subsequently by univariate logistic analysis. Factors that were found to be significantly associated with odds of carriage were included in a multivariable model to calculate the adjusted odds ratios (ORs).

\section{Ethics approval}

Ethics approval for the study was obtained from the Human Research Ethics Committee, University of Pretoria (ref. no. 268/2016).

\section{Results}

The study enrolled 322 children and infants in 2014 ( $n=96)$ and $2016(n=220)$ from two healthcare centres in Gauteng. Six children had more than five variables of demographic information missing, and their data were excluded from further analysis. Characteristics of the individuals who participated in the study and those who were colonised with S. pneumoniae are set out in Table 1.

\section{Pneumococcal serotype distribution}

Among the 121 pneumococci isolates that retained viability, 26 serotypes were detected. 19F $(10 / 121 ; 8.3 \%)$ was the most frequent PCV13 serotype. The most frequent NVTs were 23B $(16 / 121 ; 13.2 \%)$, 15B/C $(14 / 121 ; 11.6 \%)$ and 35B $(11 / 121 ; 8.2 \%)$. Overall, NVTs represented $81.8 \%$ of isolates $(21 / 26 ; 81.8 \%)$ (Fig. 1). Four carriage isolates $(4 / 121 ; 3.0 \%)$ were non-typeable by the Quellung method. There were 101 infants/children who were colonised with NVTs and 20 infants/children who carried VTs. Children aged 9 months (Fig. 2) carried the highest proportion of NVTs $(33 / 101 ; 32.7 \%)$ compared with the other ages ( 6 weeks $10.7 \%, 10$ weeks $7.4 \%, 14$ weeks $14.9 \%$, 18 months $19.8 \%, 5$ years $13.9 \%$ ) (Fig. 2). Children aged 14 weeks carried the highest proportion of VTs $(8 / 20 ; 40.0 \%)$ compared with the other ages.

\section{Risk factors associated with pneumococcal carriage}

Risk factors not included in Table 2 showed no noticeable effect on pneumococcal colonisation. In the analysis, the following risk factors were statistically insignificant: attending a day-care centre, method of cooking, and co-colonisation with $S$. aureus. Children in the age group 9 - 18 months (OR 3.4; 95\% CI 1.5 - 6.7) had an increased risk of pneumococcal colonisation compared with children aged 5 years (OR 2.8; 95\% CI 1.2 - 6.4). Children living in single-room dwellings had an increased risk of pneumococcal colonisation (OR 8.5; 95\% CI 1.3 - 52.3) compared with those living in houses, while those living in informal dwellings also had greater odds of colonisation (OR 2.4; 95\% CI 1.1 - 5.2) compared with those living in houses, keeping other factors constant (Table 2). Children colonised with $H$. influenzae had a significantly higher risk of carrying pneumococcus (OR 5.6; 95\% 


\begin{tabular}{|c|c|c|}
\hline Characteristics & $\begin{array}{l}\text { Children enrolled in the study } \\
(N=316), n(\%)\end{array}$ & $\begin{array}{l}\text { Children colonised with Streptococcus } \\
\text { pneumoniae }(N=138), n(\%)\end{array}$ \\
\hline \multicolumn{3}{|l|}{ Gender } \\
\hline Male & $158(50.0)$ & $67(48.5)$ \\
\hline Female & $147(46.5)$ & $68(49.3)$ \\
\hline Not known & $11(3.5)$ & $3(2.2)$ \\
\hline \multicolumn{3}{|l|}{ Age group } \\
\hline$\sim 6$ weeks - 14 weeks & $153(48.4)$ & $49(35.5)$ \\
\hline 9 months & $61(19.3)$ & $38(27.5)$ \\
\hline 18 months & $63(19.9)$ & $30(21.7)$ \\
\hline 5 years & $39(12.3)$ & $21(15.2)$ \\
\hline \multicolumn{3}{|l|}{ Specimen type } \\
\hline Nasopharyngeal swabs & All samples & All samples \\
\hline \multicolumn{3}{|l|}{ Vaccination } \\
\hline Yes & $267(84.5)$ & $116(84.1)$ \\
\hline No & $47(14.9)$ & $21(15.2)$ \\
\hline No vaccination records & $2(0.6)$ & $1(0.7)$ \\
\hline \multicolumn{3}{|l|}{ Previous illnesses* } \\
\hline Yes & $97(30.7)$ & $39(28.3)$ \\
\hline No & $193(61.1)$ & $92(66.7)$ \\
\hline No record & $13(4.1)$ & $7(5.1)$ \\
\hline \multicolumn{3}{|l|}{ Day-care attendance } \\
\hline Yes & $71(22.4)$ & $35(25.4)$ \\
\hline No & $232(73.2)$ & $102(73.9)$ \\
\hline No data & $13(9.4)$ & $1(0.7)$ \\
\hline \multicolumn{3}{|l|}{ Siblings $<5$ years } \\
\hline Yes & $195(61.7)$ & $86(62.3)$ \\
\hline No & $101(32.0)$ & $47(34.1)$ \\
\hline No data & $20(6.3)$ & $6(4.4)$ \\
\hline \multicolumn{3}{|l|}{ Exposure to smoking } \\
\hline Yes & $192(60.7)$ & $44(31.9)$ \\
\hline No & $115(36.4)$ & $83(60.1)$ \\
\hline No data & $9(2.9)$ & $1(7.7)$ \\
\hline
\end{tabular}

Table 2. Risk factors associated with pneumococcal carriage (univariate and multivariate analysis) in individuals from Gauteng communities, South Africa, June - December 2014 and 2015

\begin{tabular}{|c|c|c|c|}
\hline \multirow[b]{2}{*}{ Risk factors } & \multicolumn{2}{|c|}{ OR $(95 \% \mathrm{CI})$} & \multirow[b]{2}{*}{$p$-value } \\
\hline & Univariate & Multivariable & \\
\hline \multicolumn{4}{|l|}{ Age } \\
\hline $6-14$ weeks & $\operatorname{Ref}^{\dagger}$ & Ref & \\
\hline $9-18$ months & $3.5(2.0-6.2)$ & $3.4(1.9-5.9)$ & $<0.001^{\star}$ \\
\hline 5 years & $2.8(1.2-6.4)$ & $2.5(1.1-5.5)$ & $0.024^{*}$ \\
\hline \multicolumn{4}{|l|}{ Day-care attendance } \\
\hline Yes & $1.23(0.67-2.3)$ & & 0.66 \\
\hline \multicolumn{4}{|l|}{ Type of dwelling } \\
\hline Single room & $8.3(1.3-52.7)$ & $8.5(1.3-52.3)$ & $0.025^{\star}$ \\
\hline Shack (informal dwelling) & $1.8(1.0-3.3)$ & $2.4(1.2-4.5)$ & $0.010^{*}$ \\
\hline \multicolumn{4}{|l|}{ Method of cooking } \\
\hline Electric stove & 1 & & \\
\hline Paraffin stove/gas stove & $0.63(0.2-2.4)$ & & 0.501 \\
\hline Haemophilus influenzae isolated in children carrying pneumococci & $5.3(2.73-10.3)$ & $5.6(0.6-2.5)$ & $<0.001^{*}$ \\
\hline Staphylococcus aureus isolated in children carrying pneumococci & $1.2(0.68-2.4)$ & & 0.642 \\
\hline $\begin{array}{l}\text { OR = odds ratio; } \mathrm{CI}=\text { confidence interval. } \\
{ }^{*} \text { Statistically significant. } \\
\text { 'Reference, analysis based on pneumococcal carriage of } 138 \text { children. }\end{array}$ & & & \\
\hline
\end{tabular}


CI 0.6 - 2.5) compared with children who did not carry H. influenzae, also keeping other factors constant.

\section{Antimicrobial susceptibility profiles of the S. pneumoniae nasopharyngeal isolates}

Seventy-one isolates $(71 / 138 ; 51.5 \%)$ were non-susceptible to one or more antimicrobial classes using the disc diffusion method. Of the
121 serotyped isolates screened for penicillin susceptibility using oxacillin discs, $36(36 / 121 ; 29.8 \%)$ were resistant to oxacillin. Twenty NVT isolates $(20 / 36 ; 55.6 \%)$ and 2 VTs $(2 / 36 ; 5.6 \%)$ were nonsusceptible to penicillin. All the NVT (29/36; 80.5\%) and VT (7/36; $19.4 \%$ ) isolates were susceptible to cefotaxime (Table 3). Multidrug resistance was observed in $15 \mathrm{~B} / \mathrm{C}(1 / 121 ; 0.8 \%)$ and $19 \mathrm{~F}(1 / 121$; $0.8 \%)$ and 3 other pneumococcal isolates that were not serotyped

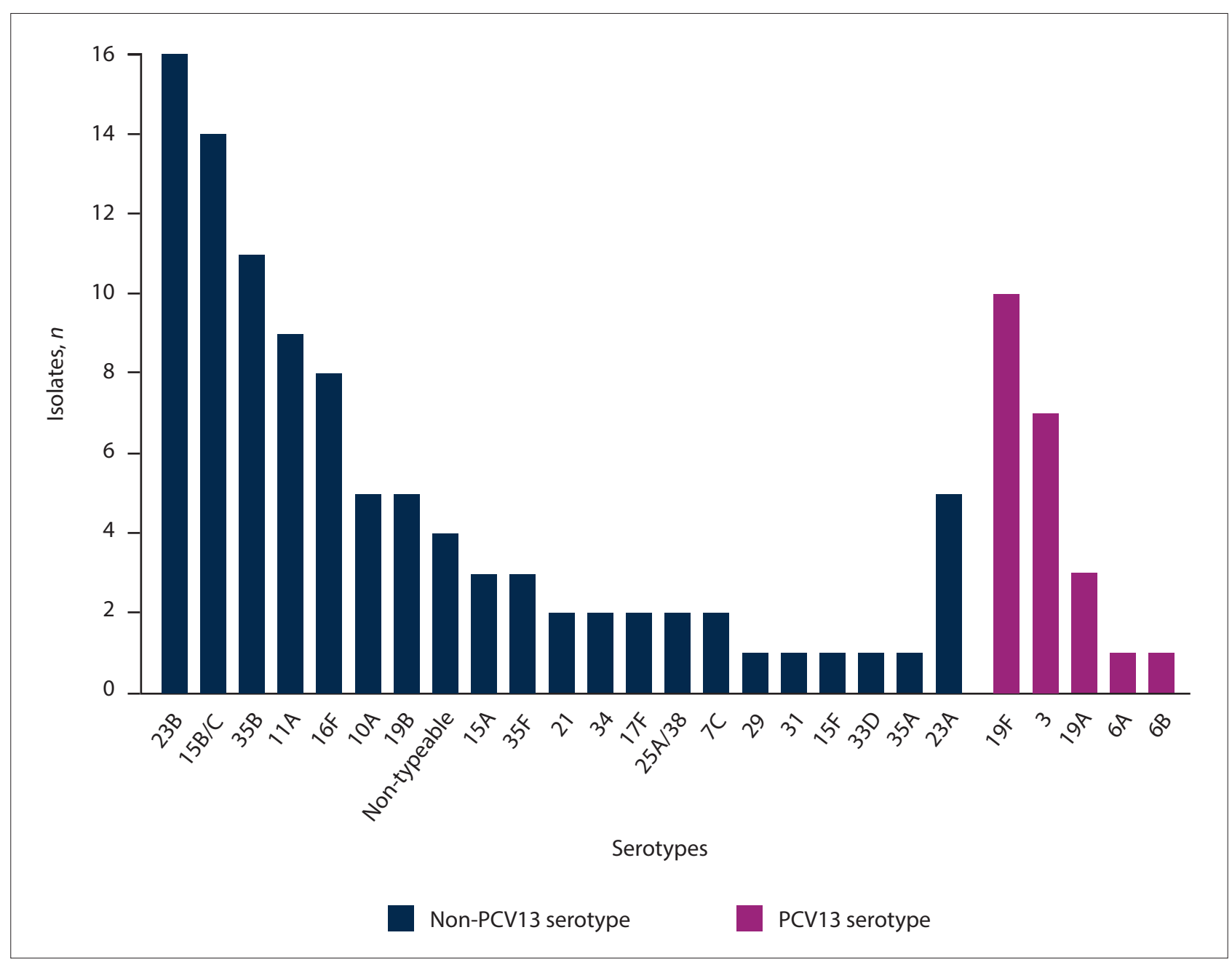

Fig. 1. Non-PCV13 and PCV13 serotypes in the children ( $\mathrm{N}=121)$ who participated in the study at two healthcare centres in Gauteng Province, South Africa, 2014 and 2016. (PCV13 = 13-valent pneumococcal conjugate vaccine).

Table 3. Antimicrobial susceptibility among the vaccine and non-vaccine serotypes of pneumococcal isolates from children presenting at two clinics in Gauteng Province, South Africa, 2014 and 2016

\begin{tabular}{|c|c|c|c|c|}
\hline \multirow[b]{3}{*}{ Antimicrobial agents } & \multicolumn{4}{|c|}{ Total $N=121, n(\%)^{*}$} \\
\hline & \multicolumn{2}{|c|}{ Non-PCV13 serotypes } & \multicolumn{2}{|c|}{ PCV13 serotypes } \\
\hline & Susceptible & Non-susceptible & Susceptible & Non-susceptible \\
\hline Penicillin $^{\dagger, *}$ & $9(25.0)$ & $20(55.6)$ & $5(13.8)$ & $2(5.6)$ \\
\hline Cefotaxime $^{\dagger}$ & $29(80.5)$ & $0(0)$ & $7(19.4)$ & $0(0)$ \\
\hline Cotrimoxazole & $55(45.5)$ & $46(38.0)$ & $12(9.9)$ & $8(6.6)$ \\
\hline Erythromycin & $95(78.5)$ & $6(8.5)$ & $18(14.9)$ & $2(2.8)$ \\
\hline Clindamycin & $98(80.1)$ & $3(4.2)$ & $20(16.5)$ & 0 \\
\hline Tetracycline & $98(80.1)$ & $3(4.2)$ & $20(16.5)$ & 0 \\
\hline MDR & \multicolumn{2}{|c|}{$1(0.8)$} & \multicolumn{2}{|c|}{$1(0.8)$} \\
\hline
\end{tabular}




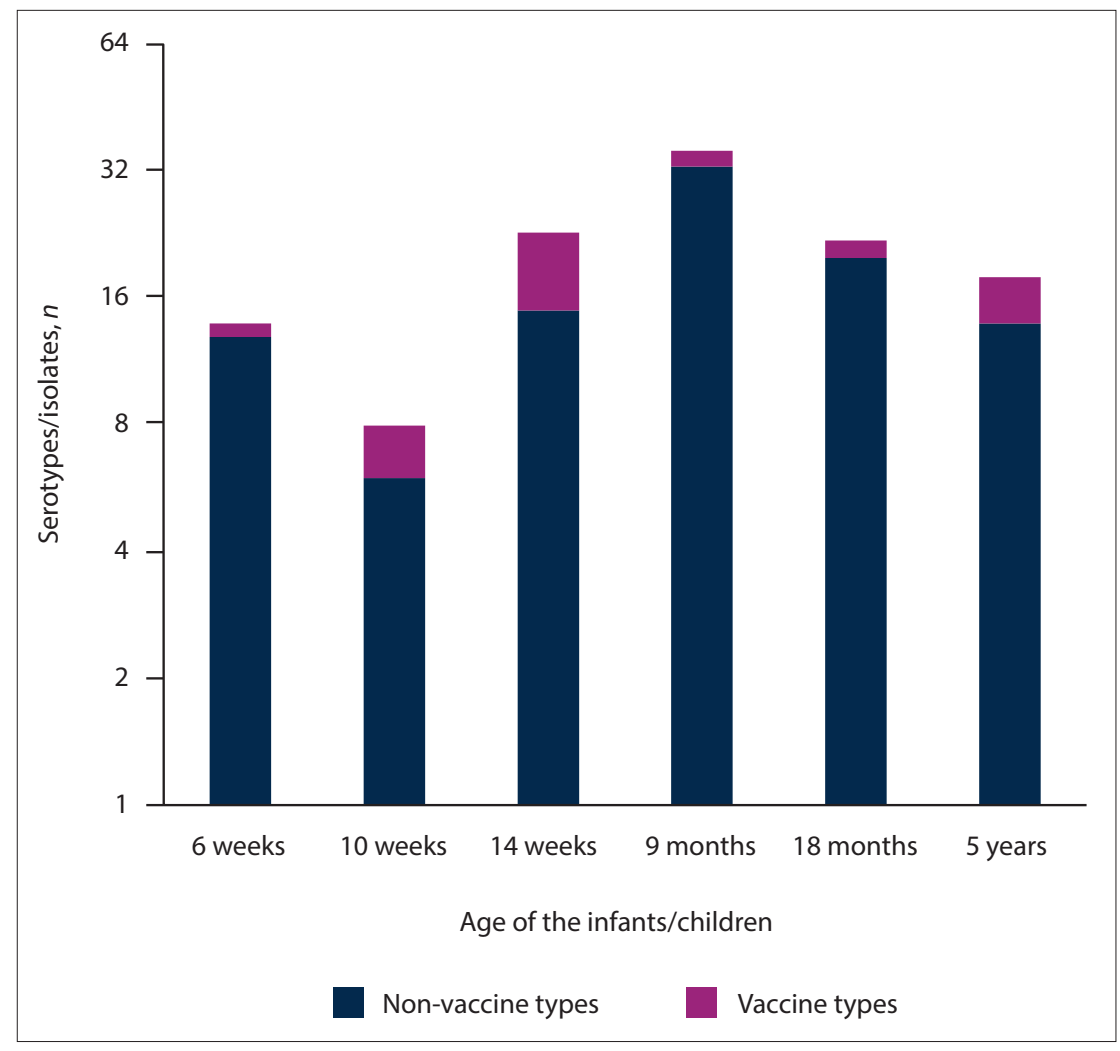

Fig. 2. Distribution of vaccine and non-vaccine serotypes in each age group of children $(\mathrm{N}=121)$ presenting for immunisation at two healthcare centres in Gauteng, South Africa, 2014 and 2016.

(isolates lost viability before they could be serotyped).

\section{Discussion}

This study reports the prevalence of pneumococcal carriage, risk factors associated with pneumococcal carriage, antimicrobial susceptibility of the carriage isolates and the common NVTs in healthy infants and children presenting for routine immunisation at two healthcare clinics in Gauteng. In our study, $43.7 \%$ of infants and children aged $\leq 5$ years were colonised with pneumococcus. This is an interesting age group in which to study nasopharyngeal carriage, because they constitute a major reservoir and source of transmission, and are at high risk of pneumococcal disease. ${ }^{[13]}$ The pneumococcal carriage prevalence obtained in this study was slightly lower compared with some carriage studies conducted in sub-Saharan Africa post-PCV. ${ }^{[3]}$ Although the reported carriage prevalence differed between the age groups of the children in our study, pneumococcal carriage usually decreases with increasing age, as has been reported in six African studies that found the youngest children to have the highest pneumococcal carriage prevalence. ${ }^{[3]}$ Other similar studies in African countries have also reported decreasing pneumococcal carriage with increasing age of healthy children. ${ }^{[3,14-16]}$
Pneumococcal carriage prevalence was high $(66.7 \%)$ in children who reported no previous illness (influenza, diarrhoea, fever, jaundice, sinusitis, meningitis or pneumonia) during the 3 months prior to their enrolment in the study. These findings contrasted with studies looking at similar populations that reported higher pneumococcal carriage in children who had respiratory tract infections during the previous 3 months. ${ }^{[16-18]}$ The low pneumococcal carriage of children reporting previous illness in our study could be attributed to recent treatment with antibiotics.

PCVs prevent pneumococcal disease by reducing pneumococcal carriage of VTs. Moreover, the impact of PCVs on nasopharyngeal carriage is considered to be a predictor of vaccine efficacy. ${ }^{[19]}$ Approximately $80 \%$ of the children who were colonised with pneumococcus had been vaccinated and had completed the specific dose for their age. Although high vaccine coverage was observed in our study, a high proportion of NVTs was reported, highlighting the importance of monitoring nasopharyngeal carriage of serotypes not included in the vaccine. In our study, the majority of children who were colonised with pneumococcus were harbouring NVTs. In addition, our study found increased NVT pneumococcal colonisation, as recently reported by other studies. ${ }^{[20,21]}$ The most common NVTs observed in this study have been reported in other studies following PCV administration. ${ }^{[22,23]}$ Dagan et al. ${ }^{[23]}$ reported NVTs (15A, 15B/C, 16F, 23A, $35 \mathrm{~B})$ with $15 \mathrm{~B} / \mathrm{C}$ significantly higher in children vaccinated with PCV13. Clinically significant NVTs identified in SA cases of IPD post PCV13 in 2014 included 8, 12F, $16 \mathrm{~F}, 35 \mathrm{~B} / 35 \mathrm{D}$ and $15 \mathrm{~B} / \mathrm{C}$, of which $35 \mathrm{~B}$ and $15 \mathrm{~B} / \mathrm{C}$ were commonly encountered in our study. ${ }^{[24]}$ Serotype $15 B$ was commonly described in our isolates, and was also among the predominant serotypes 7 years ago in an SA community study looking at temporal changes in pneumococcal colonisation of children aged $<2$ years. ${ }^{[25]}$ Some of these common NVTs identified in our study have low potential to cause disease. ${ }^{[26]}$ However, in vulnerable populations these serotypes can still cause disease. ${ }^{[24]}$ An increasing prevalence of some of these serotypes therefore warrants ongoing surveillance post vaccination. Risk factors significantly associated with pneumococcal carriage in this study included type of dwelling, young age, and carriage of $H$. influenzae. Our study did not find a significant association of day-care attendance with pneumococcal colonisation (although children attending a day-care centre had $23 \%$ higher risk compared with those who did not); however, other studies have reported that day-care centre attendance is associated with an increased risk of pneumococcal colonisation. $^{[27,28]}$ It is suggested that this increased risk is due to increased exposure to children who are colonised and even to those who may have pneumococcal disease. ${ }^{[29]}$ Among Aboriginal children and adults in Western Australia, pneumococcal carriage was significantly associated with individuals living in remote areas and younger individuals, ${ }^{[29]}$ comparable to the findings in our study. Increased pneumococcal colonisation as result of the type of dwelling (remote areas) may be attributed to exposure from the community, since these settlements are densely populated. Furthermore, the latter study also reported pneumococcal carriage associated with co-colonisation with $H$. influenzae, which was also observed in our study. In our study, a high proportion of NVTs showed non-susceptibility to penicillin. This contrasts with a study of infants at birth in Drakenstein near Cape Town, SA, in which PCV13 serotypes were more commonly non-susceptible to penicillin than NVTs. ${ }^{[20]}$ The low proportion of non-susceptible PCV13 serotypes observed may have been because of PCV, as suggested by other studies. ${ }^{[30,31]}$ 


\section{Study limitations}

The study had several limitations, including a limited sample size for comprehensively studying the epidemiology of pneumococci, and the findings obtained therefore do not reflect the overall pneumococcal carriage prevalence in the two township communities. The recruitment process may have been biased, which resulted in there being more children in the 6 - 14 weeks age group. Further studies are recommended.

\section{Conclusions}

The study found that $43.7 \%$ of enrolled children were colonised with pneumococci. Young age, type of dwelling and carrying $H$. influenzae increased the risk of being colonised with pneumococci. We also describe an increased non-PCV13 serotype pneumococcal colonisation, as has been anticipated and largely described in other studies. The increased non-PCV13 serotypes obtained from the study highlight the importance of pneumococcal colonisation studies post PCV. Of equal importance, given that pneumococcal carriage precedes disease, common colonisers such as $15 \mathrm{~B} / \mathrm{C}$ and $35 \mathrm{~B}$ may be sufficiently prevalent in carriage for expansion to result in significant disease replacement.

\section{Declaration. None.}

Acknowledgements. We thank the community health centres in Gauteng for agreeing to participate in this study, and the Chris Hani Baragwanath NHLS microbiology laboratory for their technical expertise.

Author contributions. The study was conceptualised by NM and AvG. The manuscript was drafted by ZS and revised by AvG, MdP, TM and NM. Technical guidance and analysis were provided by TA, MdP, TM and AvG. Statistical analysis was performed by SO. All authors read and approved the manuscript.

Funding. The study was funded by an NHLS Research Trust Development grant awarded to NM (grant no. 94498).

Conflicts of interest. None.

1. O'Brien KL, Wolfson LJ, Watt JP, et al. Burden of disease caused by Streptococcus pneumoniae in children younger than 5 years: Global estimates. Lancet 2009;374(9693):893-902. https://doi org/10.1016/s0140-6736(09)61204-6

2. Gray BM, Converse GM, Dillon HC. Epidemiologic studies of Streptococcus pneumoniae in infants Acquisition, carriage, and infection during the first 24 months of life. J Infect Dis 1980;142(6):923-933 https://doi.org/10.1093/infdis/142.6.923

3. Usuf E, Bottomley C, Adegbola RA, Hall A. Pneumococcal carriage in sub-Saharan Africa - a systematic review. PLoS ONE 2014;9(1):e85001. https://doi.org/10.1371/journal.pone.0085001

4. Valles X, Flannery B, Roca A, et al. Serotype distribution and antibiotic susceptibility of invasive and nasopharyngeal isolates of Streptococcus pneumoniae among children in rural Mozambique. Trop Med nasopharyngeal isolates of Streptococcus pneumoniae among children in rural Moz
Int Health 2006;11(3):358-366. https://doi.org/10.1111/j.1365-3156.2006.01565.x

5. Hill PC, Cheung YB, Akisanya A, et al. Nasopharyngeal carriage of Streptococcus pneumoniae in Gambian infants: A longitudinal study. Clin Infect Dis 2008;46(6):807-814. https://doi. in Gambian infant
org/10.1086/528688

6. La Vincente SF, von Mollendorf C, Ulziibayar M, et al. Evaluation of a phased pneumococcal conjugate vaccine introduction in Mongolia using enhanced pneumonia surveillance and community carriage surveys: A study protocol for a prospective observational study and lessons learned. BMC Public Health 2019;19(1). https://doi.org/10.1186/s12889-019-6639-y

7. Cohen R, Levy C. 13-valent pneumococcal conjugate vaccine in Africa. Lancet Glob Health 2017;5(3):e244-e245. https://doi.org/10.1016/s2214-109x(17)30044-x
8. Von Gottberg A, de Gouveia L, Tempia S, et al. Effects of vaccination on invasive pneumococcal disease in South Africa. N Engl J Med 2014;371(20):1889-1899 https://oi.org/10.1056/ nejmoa1401914

9. Nzenze SA, Madhi SA, Shiri T, et al. Imputing the direct and indirect effectiveness of childhood pneumococcal conjugate vaccine against invasive pneumococcal disease by surveying temporal changes in nasopharyngeal pneumococcal colonization. Am J Epidemiol 2017;186(4):435-444. https://doi.org/10.1093/aje/kwx048

10. Devine VT, Cleary DW, Jefferies JMC, et al. The rise and fall of pneumococcal serotypes carried in the PCV era. Vaccine 2017;35(9):1293-1298. https://doi.org/10.1016/j.vaccine.2017.01.035

11. Ubukata $\mathrm{K}$, Takata M, Morozumi M, et al. Effects of pneumococcal conjugate vaccine on genotypic penicillin resistance and serotype changes, Japan, 2010 - 2017. Emerg Infect Dis 2018;24(11):20102020. https://doi.org/10.3201/eid2411.180326

12. Clinical and Laboratory Standards Institute. M100-S24: Performance standards for antimicrobial susceptibility testing; Twenty-fourth informational supplement. January 2014. www.researchgate. et , file.PostFileLoader.html

13. Syrjänen RK, Kilpi TM, Kaijalainen TH, Herva EE, Takala AK. Nasopharyngeal carriage of Streptococcus pneumoniae in Finnish children younger than 2 years old. J Infect Dis 2001;184(4):451459. https://doi.org/10.1086/322048

14. Woolfson A, Huebner R, Wasas A, Chola S, Godfrey-Faussett P, Klugman K. Nasopharyngeal carriage of community-acquired, antibiotic-resistant Streptococcus pneumoniae in a Zambian paediatric population. Bull World Health Organ 1997;75(5):453.

15. Mbelle N, Huebner RE, Wasas AD, Kimura A, Chang I, Klugman KP. Immunogenicity and impact on nasopharyngeal carriage of a nonavalent pneumococcal conjugate vaccine. J Infect Dis 1999;180(4):1171-1176. https://doi.org/10.1086/315009

16. Arvas A, Çokuğraș H, Gür E, Gönüllü N, Taner Z, Tokman HB. Pneumococcal nasopharyngeal carriage in young healthy children after pneumococcal conjugate vaccine in Turkey. Balk Med J 2017;34(4):362-366. https://doi.org/10.4274/balkanmedj.2016.1256

17. Özdemir, H, Ciftci E, Durmaz R, et al. Nasopharyngeal carriage of Streptococcus pneumoniae in healthy Turkish children after the addition of PCV7 to the national vaccine schedule. Eur J Pediatr 2013;173(3):313-320. https://doi.org/10.1007/s00431-013-2156-7

18. Zuccotti G, Mameli C, Daprai L, et al. Serotype distribution and antimicrobial susceptibilities of nasopharyngeal isolates of Streptococcus pneumoniae from healthy children in the 13-valent pneumococcal conjugate vaccine era. Vaccine 2014;32(5):527-534 https://doi.org/10.1016/j. vaccine.2013.12.003

19. Simell B, Auranen K, Käyhty H, et al. The fundamental link between pneumococcal carriage and disease. Expert Rev Vaccines 2012;11(7):841-855. https://doi.org/10.1586/erv.12.53

20. Manenzhe RI, Moodley C, Abdulgader SM, et al. Nasopharyngeal carriage of antimicrobial-resistant pneumococci in an intensively sampled South African birth cohort. Front Microbiol 2019;10:610. https://doi.org/10.3389/fmicb.2019.00610

21. Dube FS, Ramjith J, Gardner-Lubbe S, et al. Longitudinal characterization of nasopharyngeal colonization with Streptococcus pneumoniae in a South African birth cohort post 13-valent pneumococcal conjugate vaccine implementation. Sci Rep 2018;8:12497. https://doi.org/10.1038/ 41598-018-30345-5

22. Cohen R, Levy C, Bingen E, Koskas M, Nave I, Varon E. Impact of 13-valent pneumococcal conjugate vaccine on pneumococcal nasopharyngeal carriage in children with acute otitis media. Pediatr Infect Dis J 2012;31(3):297-301. https://doi.org/10.1097/inf.0b013e318247ef84

23. Dagan R, Patterson S, Juergens C, et al. Comparative immunogenicity and efficacy of 13 -valent and 7-valent pneumococcal conjugate vaccines in reducing nasopharyngeal colonization: A randomized double-blind trial. Clin Infect Dis 2013;57(7):952-962. https://doi.org/10.1093/cid/cit428

24. Lo SW, Gladstone RA, van Tonder AJ, et al. Pneumococcal lineages associated with serotype replacement and antibiotic resistance in childhood invasive pneumococcal disease in the postPCV13 era: An international whole-genome sequencing study. Lancet Infect Dis 2019;19(7):759769. https://doi.org/10.1016/S1473-3099(19)30297-X

25. Nzenze SA, Shiri T, Nunes MC, et al. Temporal changes in pneumococcal colonization in a rural African community with high HIV prevalence following routine infant pneumococcal immunization. Pediatr Infect Dis J 2013;32(11):1270-1278. https://doi.org/10.1097/01.inf.0000435805.25366.64

26. Lindstrand A, Galanis I, Darenberg J, et al. Unaltered pneumococcal carriage prevalence due to expansion of non-vaccine types of low invasive potential 8 years after vaccine introduction in Stockholm, Sweden. Vaccine 2016;34(38):4565-4571. https://doi.org/10.1016/j.vaccine.2016.10.050

27. Huang SS, Finkelstein JA, Lipsitch M. Modeling community- and individual-level effects of childcare center attendance on pneumococcal carriage. Clin Infect Dis 2005;40(9):1215-1222. https:// doi.org/10.1086/428580

28. Koliou MG, Andreou K, Lamnisos D, et al. Risk factors for carriage of Streptococcus pneumoniae in children. BMC Pediatr 2018;18(1):144. https://doi.org/10.1186/s12887-018-1119-6

29. Collins DA, Hoskins A, Snelling T, et al. Predictors of pneumococcal carriage and the effect of the 13-valent pneumococcal conjugate vaccination in the Western Australian Aboriginal population. Pneumonia 2017;9:14. https://doi.org/10.1186/s41479-017-0038-x

30. Klugman KP, Madhi SA, Huebner RE, Kohberger R, Mbelle N, Pierce N. A trial of a 9-valent pneumococcal conjugate vaccine in children with and those without HIV infection. N Engl J Med 2003;349(14):1341-1348. https://doi.org/10.1056/nejmoa 035060

31. Tomczyk S, Lynfield R, Schaffner W, et al. Prevention of antibiotic-nonsusceptible invasive pneumococcal disease with the 13 -valent pneumococcal conjugate vaccine. Clin Infect Dis 2016;62(9):1119-1125. https://doi.org/10.1093/cid/ciw067

Accepted 8 June 2020 